\title{
Estructura de control y sus correlatos en una burocracia profesional: el caso de un hospital
}

\author{
FÉLIX PÉREZ QUINTANA \\ Universidad de La Laguna
}

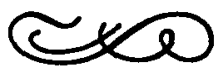

Resumen

Se utilizó el método del a Gráfico de Controls de Tannenbaum con el fin de estudiar la estructura de poder y autoridad de una organizaciön hospitalaria. Los resultados obtenidos confirman el carácter de burocracia profesional característico de este tipo de organizaciones. Se detectó una disminución del control percibido de la linea administrativa por parte del componente más profesionalizado. Asi mismo se demostró la percepción diferencial de la estructura de control por parte de los miembros en función de su localización estructural y sus efectos sobre el contexto diseño y eficacia de los puestos y unidades, confirmándose los resultados de otros estudios que ban detectado este mismo fenómeno. Estos resultados apoyan la bondad "Gráfico de Controls para el estudio de la estructura de poder $y$ autoridad en las organizaciones formales.

\section{Abstract}

The Tannenbaum's \&Control-Graph, method was used with the purnnce of nnalyring the distributzon of power and authority of an health care organization. The obtained results confirm the nature of profesional bureaucracy characteristic of this type of organization. It was detected a decrease of the perceived control of the administrative line on part of the most professionalized component. The differential perception of control structure was demonstrated in the same way on part of the members, depending on its structural location and on its effects on the design, context and efficacy of the jobs and units, being confirmed other inverstigation's results which have detect the same phenomenon. This results support the goodness of Control-Graph method for the study of power and authority in formal organizations.

Dirección del autor: Facultad de Filosofía y Ciencias de la Educación. Universidad de La Laguna. Tenerife. 


\section{INTRODUCCION}

El problema del control ha sido, en las últimas décadas, un tema recurrente en la teoría e investigación de las organizaciones. La razón de este interés está en la importancia que para las mismas tiene la reducción de la variabilidad personal de los miembros que las componen en orden a la consecución de las metas globales. Esta variabilidad tiene como fuentes tanto las aptitudes individuales como las actitudes, valores, intereses, cultura, orígenes, metas personales, o la diferenciación de los procesos grupales formales e informales.

Las formas de control más usuales se basan de una parte en los procesos de normalización, formalización y regularización de las actividades y de otra en el ejercicio del poder, la autoridad o la influencia.

Diversos autores (p.e. Minztberg, 1984) han señalado la importancia que el diseño de esta estructura de control tiene en aquellas organizaciones que, como la que es objeto de nuestro trabajo, existe un alto componente profesional en la línea principal de actividad laboral. Esto justifica el interés del estudio de los correlatos de dicha estructura con los patrones de diseño de los puestos y unidades o que conforman la división del trabajo en la organización.

Estos cuatro términos: control, poder, autoridad e influencia constituyen, desde el punto de vista teórico y conceptual, procesos diferentes.

Una de las definiciones más frecuentes del poder hace referencia a la capacidad de un actor $A$ para obtener de otro $B$ un cambio o actuación determinada (Filley et al., 1976). Entre las clasificaciones teóricas más conocidas que han intentado operacionalizar esta definición amplia de poder se encuentran la de Etzioni (1961), que considera tres formas: coercitiva, remunerativa y normativa; y la ampliamente difundida de French y Raven (1959) que establece cinco bases del poder: la coercitiva, de recompensa, legítima, referente y de experto.

La distinción entre poder y autoridad ha sido establecida, de acuerdo con Weber (1979), entendiendo que en tanto que el poder es la capacidad para actuar, la autoridad es el derecho a actuar. Por tanto la autoridad quedaría reducida, desde este punto de vista, al ejercicio del poder legítimo, normativo o posicional (Zey-Ferrell, 1979).

Por influencia se entiende, siguiendo a Presthus (1962), la capacidad de A para obtener un resultado de B sin acudir al recurso de recompensas o sanciones. Esto es, la influencia puede darse sin que exista poder o autoridad (Filley et al., 1976).

Finalmente, el término control ha pasado a ser utilizado por un buen número de autores, para referirse al sistema de integración y coordinación de actividades que tiene como base fundamental el ejercicio del poder, la autoridad y la influencia. Este proceso de control no se da necesaria y únicamente en la dimensión vertical y jerárquica del sistema, sino también en la horizontal y espa. cial; y en todos los niveles: personal, grupal y global. (Rai, 1986; Rabbie y Van Oostrum, 1984).

\section{CONSIDERACIONES SOBRE EL CONTROL Y LA EFICACIA}

A pesar del evidente interés y utilidad de esta diferenciación teórica. estos procesos han sido considerados, desde el punto de vista práctico y empírico. como sinónimos. Existe el consenso de que en la prácrica los miembros de una organización no distinguen entre poder, autoridad e influencia a la hora de des- 
cribir los procesos de control que funcionan en la misma (Rai, 1985; Van de Ven y Ferry, 1980).

Otro factor que afecta al ejercicio y estudio del control en las organizaciones es la percepción del mismo por parte de los miembros. El organigrama y el régimen o reglamento interno representan las intenciones de control interpersonal que se desean establecer pero, sin embargo, en la práctica la autoridad no existe a menos que sea percibida como tal por los miembros. Esto es, la comparación entre la estructura formal y la percepción de la autoridad puede dar indicios de en qué medida la estructura de control planificada o propuesta se desvía de los patrones de relación de autoridad realmente existentes en la organización (Van de Ven \& Ferry, 1981).

En orden al estudio del efecto de la estructura de control sobre el desempeño y eficacia de la organización, una de las clasificaciones más utilizadas ha sido la distinción entre estructuras centralizadas y descentralizadas. Simon (1954, cfr. Jennergren, 1981), define la centralización como:

«... la medida en que las decisiones se toman en niveles relativamente altos de la organización; la descentralización se da en la medida en que la discrecionalidad y la autoridad para tomar decisiones importantes se delegan a niveles inferiores de autoridad» (Pág. 39).

Otras definiciones de centralización hacen referencias más concretas al grado en que los supervisores consultan a los subordinados sobre las decisiones a tomar en la unidad y sobre el establecimiento de las metas y objetivos de la misma (cfr. Zey-Ferrell, 1979); o a la discrecionalidad de estos para tomar decisiones relacionadas con su propio trabajo (Van de Ven \& Ferry, 1980).

El control se ejerce esencialmente a través de procesos de toma de decisión que, si bien son necesarios cara al mantenimiento del sistema, también suponen un consumo de recursos y energía, de tal modo que una utilización inadecuada de los mismos pueden llevar a disfunciones, tales como la rigidez estructural y funcional (Jawler, 1976), desmotivación, baja en la motal de los compleados, conflicto, etc. (Jennergren, 1981).

La bibliografía publicada que atañe a este efecto del patrón de control sobre la eficacia organizacional y los resultados personales así como a la adaptatividad del sistema a las condiciones ambientales ha sido abundante. En ella se han planteado diversas hipótesis y resultados derivados de la investigación, en oca. siones contradictorios.

A modo de ejemplo tenemos las hipótesis derivadas de la teoría axiomática de Hage (1965), que postula una mayor eficacia asociada al incremento de la centralización. Y como contraste tenemos las propuestas formuladas por Tannenbaum (1968), que asocian el incremento de la eficacia a la descentralización.

Los resultados personales tales como la moral, satisfacción y motivación han aparecido positivamente asociados de modo más consistente con la descentralización (Khandwalla, 1977; Tannembaum, 1968; Lorch, 1978).

En cuanto a la adaptatividad tampoco se han generado investigaciones o hipótesis que puedan ser aceptadas de modo definitivo. Así, se ha encontrado que la descentralización mejora la capacidad de adaptación en la medida que los decisores locales o departamentales pueden responder a los cambios ambien. tales sin depender de las decisiones de sus superiores (Hage, 1965; Price, 1968). Pero otros autores como Blau y Schoenherr (1971), encuentran que la centralización puede facilitar la adaptación en la medida en que las decisiones dependen de un pequeño grupo, la alta gerencia, reduciéndose de este modo la depen- 
dencia del consenso entre un gran número de personas. Una tercera alternativa es la que proponen autores como Duncan (1976) y Zaltman et al., (1973), que postulan la bondad de la estructura centralizada para llevar adelante cambios predeterminados y la de la descentralizada para la inicialización de cambios no previstos. Jennergren (1981), en una revisión sobre este tema concluye que el impacto de la descentralización sobre la adaptatividad no está aún claramente establecido.

\section{CONTROL Y BUROCRACIAS PROFESIONALES}

Desde el punto de vista taxonómico y siguiendo la clasificación de Katz y Kahn (1966), las organizaciones hospitalarias desempeñan funciones genotípicas de mantenimiento y sus procesos están centrados en las personas. En otras palabras el producto principal de un hospital es el cuidado y restauración de la salud de los pacientes. La calidad de este producto, por su propia naturaleza, no puede ser totalmente predicha ni estandarizada.

Sin embargo, comparten ciertas características con otros tipos generales de organizaciones, fundamentalmente con las adaptativas y productivas. Con las primeras comparten la actividad investigadora y de generación de conocimientos; y con las segundas, especialmente con las industriales, el uso de procedimientos que implican la utilización de alta tecnología, el énfasis en la prevención de errores y una elaboración rígida de roles y normas de actuación con el fin de garantizar un desempeño correcto y eficaz de las tareas, que evite consecuencias que pueden ser fatales para la salud y la vida de los pacientes (Katz y Kahn, 1966; Kouzes y Mico, 1979).

Otra característica definitoria de los hospitales, que comparten con otras organizaciones con metas centradas en el servicio a personas, es la existencia de tres dominios diferenciados en cuanto a su legitimación, disposición estructural y modos de trabajo: El Dominio Político, en el que se formulan los procedimientos de gobierno y la política global a seguir; el Dominio de Gestión, responsable de maximizar los beneficios para el sistema, siguiendo, generalmente, el modelo de gestión de las organizaciones industriales, centrado en el control administrativo; y el Dominio de Servicios, que se encarga directamente del cuidado y restauración de la Salud (Kouzes y Mico, 1979).

En este último dominio actúan personas (médicos y enfermeras) cuya legitimación para el desempeño de las tareas viene dada por una capacitación adquirida tras un largo período de formación. Los principios de gobierno y control de este dominio se basan en la autonomía y la autorregulación y los criterios de eficacia se basan, generalmente, en la «calidad del servicio» o en «criterios profesionaless. Estos criterios suelen referirse a procesos y no a productos. El modo de trabajo que se adopta es individualizado, basado en la solución de los problemas particulares de cada cliente y con la utilización de una tecnología en buena medida inespecífica.

Este alto grado de profesionalización en la línea principal de actividad, en contraste con la industria donde los profesionales tienden a concentrarse en el «staff», afecta de modo diverso a la estructura y contexto de la organización.

La forma en que el incremento en el índice de profesionalización de la línea principal de actividad afecta a la estructura de control ha sido abundantemente estudiada, de tal modo que se ha acuñado un término - burocracia profesional (Minztberg, 1984)- para referirse a este tipo de sistemas. 
La coexistencia de tres líneas diferenciadas de autoridad: la política, la gerencial y la profesional, plantea problemas complejos en el reparto de la influencia y el poder y por tanto en la capacidad de toma de decisiones sobre los diferentes eventos de la actividad laboral.

En la práctica tienden a coexistir dos estructuras paralelas: una descendente de tipo maquinal basada en la autoridad formal, en la que se integra el personal administrativo de apoyo y de gestión; y otra ascendente de tipo profesional que tiende a utilizar procedimientos de toma de decisión más participativos y democráticos.

Esta coexistencia puede suponer un choque entre las metas racionalizadoras y optimizadora de beneficios de la línea administrativa y las metas de eficacia cualitativa y profesional de la línea médica. La discrepancia puede nutrirse asimismo de una diferencia de valores e ideología entre los componentes de ambas líneas.

Las fuentes sobre las que se basa el poder y la influencia de los profesionales son lo suficientemente relevantes para la organización como para suponer un constante juego de poderes en orden a la prevalencia de uno u otro tipo de metas o intereses.

De una parte se encuentra el nivel de experto de los profesionales que no permite prescindir de ellos o sustituirlos sin que se vea afectada la calidad del servicio. A este nivel de experto, derivado de una formación larga y costosa, hay que añadir el efecto del uso de nuevas y avanzadas técnicas de diagnóstico y tratamiento, que en la medida en que solamente es el componente más profesionalizado el que las comprende y domina se transforman en una fuente de poder para el mismo (Fineberg, 1985).

Así, por ejemplo, se ha encontrado que en hospitales altamente tecnificados y con unidades muy especializadas una buena parte de las decisiones operativas las llevan a cabo los directores de las unidades; esto supone una descentralización vertical y un anmento de la influencia y el poder en el rango medio do la organización (Overton, 1977).

Otros autores (Cfr. Peiró, 1984: 295), han encontrado que en organizaciones altamente profesionalizadas la estandarización y formalización de las tareas correlaciona positivamente con la centralización, en tanto que la formalización de normas globales para el conjunto de la organización lo hace negativamente.

Por otra parte la existencia de un alto componente profesional supone un cierto grado de control externo por parte de las organizaciones profesionales que tratan de asegurarse el control de sus miembros mediante la exigencia de la colegiación, el establecimiento de códigos éticos y la administración de sanciones a los miembros que se desvían de sus normas (Flood y Sott, 1978).

Todo esto supone en muchas ocasiones una dificultad extra para la gerencia en el control del trabajo y la corrección de las deficiencias que los propios profesionales decidan pasar por alto.

La importancia que este componente da al control sobre sus propias actividades en detrimento del control ejercido por la línea administrativa se plasma en los resultados de diversas investigaciones que manifiestan que un aumento del poder departamental y profesional aparece generalmente ligado a un aumento de la eficacia de las unidades (Cfr. Flood y Scott, 1978).

En la medida en que este conflicto entre los distintos dominios y el aumento de la capacidad de toma de decisión en el rango medio plantea problemas graves en la integración del sistema, se ha tratado de implantar diseños alternati- 
vos que permita obviarlos. Uno de los más socorridos han sido los diseños matriciales en los que en lugar de que el responsable de cada área tome las decisiones que le afectan de forma independiente y a través de una única línea de mando se trata de compartir y descentralizar las decisiones entre los responsables de áreas distintas sacrificando el principio de unidad de mando. Sin embargo esta alternativa ha presentado dificultades, en ocasiones difíciles de salvar. Entre éstas se encuentran la incertidumbre generada por la duplicación de la línea de mando que se convierte en fuente de stress, no sólo para los directivos para los que representa inseguridad y conflicto, sino también para los subordinados. La presencia de más de un superior para un individuo, la vaguedad en las instrucciones, y el número de demandas en procesos destinados a la planificación y gestión pueden dar lugar a conflictos, ambigüedades y sobrecarga de roles (Mintzberg, 1984).

\section{LA MEDIDA DEL CONTROL. EL GRAFICO DE CONTROL DE TANNENBAUM}

Los procedimientos e indicadores utilizados para medir la distribución de poder e influencia son diversos. La mayor parte de ellos se basan en las características del organigrama formal, como por ejemplo el campo de control de los supervisores (Blau y Scott, 1962), el número de niveles de supervisión o la razón supervisores/staff (Melman, 1958).

La teoría del gráfico de control de Tannenbaum (1968), ha aportado un procedimiento basado en cuestionarios fácilmente aplicables, que ha recibido un amplio apoyo desde la investigación.

Mediante este método el grado de control percibido se mide preguntando a los miembros de los diversos niveles «la cantidad de influencia o autoridad» que tiene cada grupo (p.e. gerente, jefes de departamento, supervisores de primer nivel, etc.), incluido el propio, «para decidir lo que se hace en la organización». La influencia o control percibido de cada grupo se calcula promediando las respuestas dadas bien por todos los miembros, obteniéndose un gráfico global, o por los pertenecientes a cada nivel, con lo que se obtiene un gráfico para cada nivel. Estos datos se representan en un sistema de coordenadas en el que el eje horizontal representa los distintos grupos y el vertical la cantidad de influencia percibida para cada uno de ellos.

A partir de este gráfico se pueden estimar dos aspectos de la estructura de control: (1) La cantidad de control ejercido por los miembros de cada grupo, representado por el valor en ordenadas y (2) La distribución del control representada por la forma o pendiente del gráfico. Este último concepto es semejante al de centralización utilizado por los autores anteriormente comentados. Por regla general la pendiente del gráfico es negativa reflejando una mayor cantidad de control en los niveles altos de la organización, reduciéndose a medida que se baja en los niveles jerárquicos. Cuanto más negativa es la pendiente mayor será el grado de centralización.

Aunque el método del gráfico de control ha recibido algunas críticas, que afectan, por ejemplo, a su validez interna en la medida en que cada persona entiende de modo distinto el concepto de poder, o a la consideración unidimensional del índice (Gundelach y Tetzschner, 1976), este sistema cuenta con numerosos defensores y datos que apoyan su utilidad en la medida en que permi- 
te la descripción de los grupos y organizaciones a nivel de sistema y no de individuos (Rabbie y Van Oostrum, 1984).

Otra premisa teórica en la que se apoya el gráfico de control es que la consideración clásica de que hay una cantidad fija de poder a repartir, de modo que el aumento en control de un grupo supone el decremento de otros no se considera válida. Se entiende que el reparto de influencia o control no es un juego de suma cero, en el que el aumento de influencia de un grupo se hace a costa de la pérdida de la de otros, de tal modo que se puede dar un incremento en la capacidad decisoria de un grupo sin que esto afecte a la de los demás (Tannenbaum, 1968).

El objetivo de esta investigación ha sido probar la bondad del método del gráfico de control para la medida de la estructura de control de una organización hospitalaria. Asimismo nos hemos propuesto explorar los correlatos de la estructura percibida de control con algunos índices del contexto, diseño y resultados de los puestos y unidades de trabajo.

\section{METODO}

\section{La Organización estudiada}

La organización en la que se llevó a cabo este estudio es el Hospital Infantil de Santa Cruz de Tenerife. En él trabajan 126 personas de las que 29 son Médicos, 12 Enfermeras, 37 Auxiliares Clínicos, y el resto forma parte del personal de administración y servicios. De este personal, 37 son varones y 89 mujeres. Cuenta con una capacidad de 100 camas utilizables, distribuidas en diez salas. Está dotado con los servicios necesarios para atender a la población infantil en todos los aspectos sanitarios que no requieran cirugía mayor o procedimientos de alta tecnolngía. Tiene tres divisiones principales: la de Scrivicius Geñé̃äies que incluye el personal de cocina, limpieza y mantenimiento: la de administración y la médica que incluye todos los servicios dedicados a la atención sanitaria de los pacientes ingresados y de los que acuden a las consultas externas.

\section{Sujetos}

Participaron en el estudio 118 personas, lo que representa el $94 \%$ del total del personal del Hospital. De estos, 86 fueron mujeres y 31 varones. Durante el proceso de recogida de datos dos de las personas, que inicialmente se habían prestado a participar, entregaron los cuestionarios sin cumplimentar. Por niveles, las personas que contestaron se distribuyen de la siguiente manera: $75 \mathrm{em}$ pleados de primera línea, 9 enfermeras supervisores de primera línea, 24 médicos responsables de servicios o especialistas, y 3 del nivel de la Direccion-Gerencia. Por divisiones, 25 pertenecen a la división de servicios generales, 15 a la de administración, y a la médica 75 . La edad de los encuestados, cubre un rango de 21 a 62 años, con una media de 37 y desviación típica de 10 . En cuanto a la antigüedad en el hospital, la media se encuentra entre los 6 y 10 años de permanencia en el mismo. Por nivel educativo: 51 poseen el Graduado Escolar o la EGB, 14 el Bachiller, 11 títulos de Formación Profesional, 13 de Graduado Universitario y 25 de Licenciado y 1 de Doctor. 


\section{4}

\section{Instrumentos}

Se utilizó el procedimiento del gráfico de Control de Tannenbaum para la medida de la estructura de control. Al método utilizado por Tannenbaum se le hicieron las siguientes modificaciones:

1) Puesto que el control se puede repartir no sólo entre los componentes específicos de la jerarquía sino también entre otras unidades del mismo nivel y personas ajenas a la organización se amplió el eje de abcisas con el fin de recoger estos elementos.

2) El método propuesto por Tannenbaum (1968), utiliza una única pregunta general. Dado que en diferentes estudios se han encontrado diferencias sistemáticas en función de la actividad o proceso organizacional involucrado, hemos planteado preguntas específicas acerca del grado de influencia de cada grupo distinguiendo las distintas áreas sobre la que se ejerce dicha influencia.

Se planteó la pregunta general: «¿Qué cantidad de influencia piensa que tienen cada una de las siguientes personas...?, en tres cuestionarios diferentes. En uno la pregunta hacia referencia a las operaciones y funciones internas de la unidad, en el segundo a todo el hospital y el tercero a los eventos o actividades externas. Los grupos o personas respecto a los que se planteó la cuestión fueron: los miembros de las unidades, el supervisor, otros supervisores de otras unidades, el director médico, el gerente, la jefa de personal, el comité de empresa, y personas ajenas al hospital. Se urilizó una escala con los cinco anclajes siguientes: «Nada», «Poco», «Algo», «Bastante», «Mucho».

Los índices de contexto, diseño y resultados de los puestos se midieron utilizando los módulos respectivos de la versión española del juego de escalas para la evaluación organizacional (OAI) de Van de Ven y Ferry (1980, Cfr. Pérez Quintana, 1987).

\section{RESULTADOS}

\section{Estructura percibida de control}

Con el fin de hallar el gráfico de control tanto global como por niveles y unidades se calcularon las medias y desviaciones típicas en función de cada uno de estos factores estructurales para cada una de las áreas de influencia.' La pendiente de cada gráfico se estimó promediando las diferencias algebraicas entre las cantidades de control absoluto resultante para cada uno de los niveles, método utilizado por Tannenbaum (1968), y que han seguido posteriormente diferentes autores (Bower, 1968; Gundelach y Tetzschner, 1976; Rai, 1985).

\section{a) Control por áreas}

La tabla I muestra las medias y desviaciones típicas de los índices de control sobre las distintas áreas para cada uno de los grupos evaluados. También contiene el valor del índice global resultante de promediar los correspondientes a las tres áreas de influencia. La figura 1 muestra los gráficos correspondientes a las tres áreas de influencia.

Las, pendientes para cada uno de estos gráficos son, respectivamente: Unida$\operatorname{des}(P=-.34)$; Hospital $(P=-.34))$ y Eventos Externos $(P=-.30)$. Este resulta- 
TABLA I

Medias y desviaciones típicas de los índices de control percibido

\begin{tabular}{|c|c|c|c|c|c|}
\hline Indices & Media & $\begin{array}{l}\text { Desv. } \\
\text { típica }\end{array}$ & Indices & Media & $\begin{array}{l}\text { Desv. } \\
\text { típica }\end{array}$ \\
\hline $\begin{array}{l}\text { 1. Control medio de los miembros } \\
\text { de las unidades } \\
\text { a. Sobre las operaciones de la } \\
\text { unidad } \\
\text { b. Sobre todo el hospital } \\
\text { c. Sobre enventos y circunstan- } \\
\text { cias externas } \\
\text { 3. Control medio de supervisores } \\
\text { de otras unidades } \\
\text { a. Sobre las operaciones de la } \\
\text { unidad } \\
\text { b. Sobre todo el hospital } \\
\text { c. Sobre eventos y circunstan- } \\
\text { cias externas } \\
\text { 5. Control medio del gerente } \\
\text { a. Sobre las operaciones de la } \\
\text { unidad } \\
\text { b. Sobre todo el hospital } \\
\text { c. Sobre eventos y circunstan- } \\
\text { cias externas } \\
\text { 7. Control medio del comité de } \\
\text { empresa } \\
\text { a. Sobre las operaciones de la } \\
\text { unidad } \\
\text { b. Sobre todo el hospital } \\
\text { cobre eventos y circunstan- } \\
\text { cias externas }\end{array}$ & $\begin{array}{l}1.98 \\
3.44 \\
3.85 \\
2.42 \\
2.22 \\
4.27 \\
3.78 \\
4.57 \\
4.47 \\
2.23 \\
1.65 \\
2.48 \\
2.57\end{array}$ & \begin{tabular}{|l|}
0.84 \\
1.26 \\
1.16 \\
0.84 \\
\\
0.82 \\
1.27 \\
1.19 \\
1.19 \\
1.44 \\
0.73 \\
1.17 \\
1.37 \\
0.88 \\
\end{tabular} & $\begin{array}{l}\text { 2. Control medio de supervisores } \\
\text { de otras unidades } \\
\text { a. Sobre las operaciones de la } \\
\text { unidad } \\
\text { b. Sobre todo el hospital } \\
\text { c. Sobre eventos y circunstan- } \\
\text { cias externas } \\
\text { 4. Control medio del director mé- } \\
\text { dico } \\
\text { a. Sobre las operaciones de la } \\
\text { unidad } \\
\text { b. Sobre todo el hospital } \\
\text { c. Sobre eventos y circunstan- } \\
\text { cias externas } \\
\text { 6. Control medio de la jefa de } \\
\text { personal } \\
\text { a. Sobre las operaciones de la } \\
\text { unidad } \\
\text { b. Sobre todo el hospital } \\
\text { c. Sobre eventos y circunstan- } \\
\text { cias externas } \\
\text { 8. Control medio de personas aje- } \\
\text { nas al hospital } \\
\text { a. Sobre las operaciones de la } \\
\text { unidad } \\
\text { b. Sobre todo el hospital } \\
\text { c. Sobre eventos y circunstan- } \\
\text { cias externas }\end{array}$ & $\begin{array}{l}2.86 \\
3.44 \\
2.92 \\
2.22 \\
\\
3.49 \\
\\
2.73 \\
3.92 \\
3.85 \\
\\
3.03 \\
3.08 \\
3.43 \\
2.57 \\
1.99 \\
1.37 \\
2.14 \\
2.44\end{array}$ & $\begin{array}{l}0.99 \\
1.27 \\
1.31 \\
1.44 \\
0.91 \\
1.40 \\
1.06 \\
1.19 \\
0.95 \\
1.28 \\
1.06 \\
1.16 \\
1.10 \\
0.96 \\
1.44 \\
1.51\end{array}$ \\
\hline
\end{tabular}

FIGURA 1

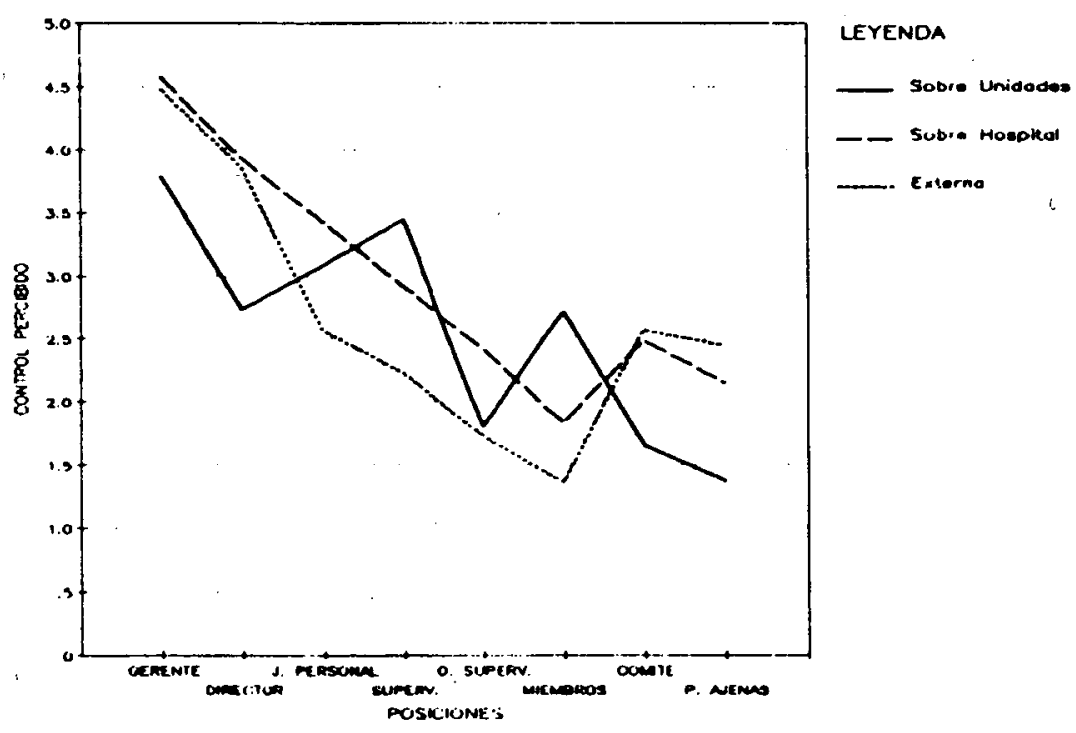

Influencia percibida de las posiciones jerárquicas por áreas de influencia. 
do señala una distribución de control semejante en las decisiones que afectan a las unidades y a todo el hospital y ligeramente inferior en el área de las relaciones externas donde la centralización parece menor incrementándose en esta la influencia del comité de empresa y naturalmente la de personas ajenas a la organización.

La cantidad y distribución del control varía en función del área de influencia considerada. La estructura que hace referencia a la influencia sobre las actividades globales del hospital es la que muestra un decremento más sistemático en función de la posición jerárquica de los distintos grupos o cargos evaluados. Se aprecia cómo la influencia de los supervisores y de los miembros sufre un incremento relativo en el ámbito de las unidades en tanto que disminuye de forma considerable en lo que atañe a los eventos externos y al hospital en general.

La cantidad de control de los miembros es muy baja en todos los ámbitos. La puntuación más alta la alcanzan en el área de las unidades; en las restantes las puntuaciones corresponden a las categorías «nada» y «poco». El gerente aparece con mayor cantidad de control que el director médico en todas las áreas. La tabla I muestra que la dispersión en cuanto a la influencia de cada grupo o posición en las distintas áreas es notable dándose la mayor discrepancia en el control percibido del director médico y el gerente.

\section{b) Control Global}

La tabla II muestra los coeficientes de correlación entre los índices de influencia en cada área de cada uno de los grupos o posiciones, así como el coeficiente $\alpha$ de Cronbach correspondiente. Los valores de estos coeficientes, aun cuando alguno de ellos tienden a ser bajos, avalan la posibilidad de calcular un índice

Figura 2

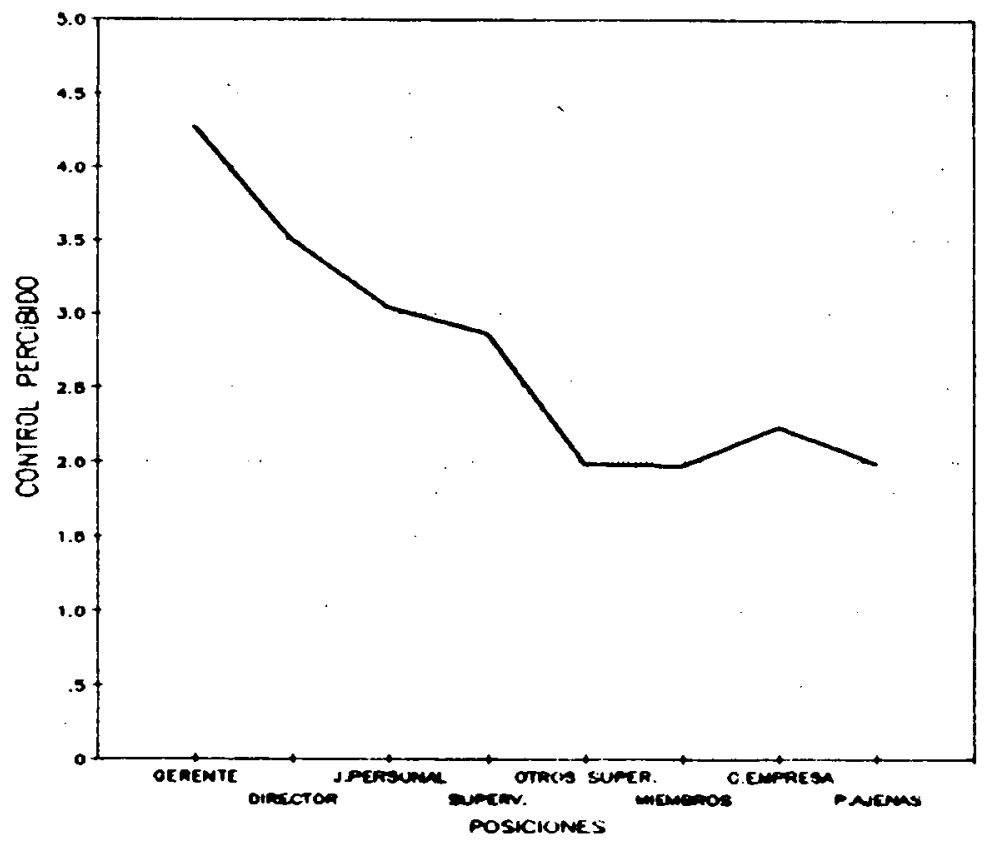

Gráfico del control percibido global para todas las posiciones. 
global para cada grupo o posición en la medida en que las correlaciones de las puntuaciones globales con las de las distintas áreas son bastante estimables cubriendo un rango de $\mathrm{t}=.57$ a $\mathrm{r}=.88$.

\section{TABLA II}

Correlaciones entre las áreas de decisión dentro de cada indice global de control y coeficiente Alpha correspondiente

\begin{tabular}{|c|c|c|c|c|c|c|c|c|c|}
\hline \multirow{2}{*}{ Indices } & \multicolumn{4}{|c|}{ Area de decisión } & \multirow{2}{*}{ Indices } & \multicolumn{4}{|c|}{ Area de decisión } \\
\hline & Unid. & Hosp. & Ext. & c & & Unid. & Hosp. & Ext. & c \\
\hline $\begin{array}{l}\text { 1. Control medio de los } \\
\text { miembros de las unida- } \\
\text { des } \\
\text { a. Sobre las operaciones } \\
\text { de la unidad } \\
\text { b. Sobre todo el hospital } \\
\text { c. Sobre eventos y cir- } \\
\text { cunstancias externas }\end{array}$ & .78 & $\begin{array}{l}.82 \\
.43 \\
-\end{array}$ & $\begin{array}{l}.27 \\
.45\end{array}$ & .64 & $\begin{array}{l}\text { 2. Control medio de los su- } \\
\text { pervisores de las unida- } \\
\text { des } \\
\text { a. Sobre las operaciones } \\
\text { de la unidad } \\
\text { b. Sobre todo el hospital } \\
\text { c. Sobre eventos y cir- } \\
\text { cunstancias externas }\end{array}$ & - & $\begin{array}{c}.86 \\
.30 \\
-\end{array}$ & $\begin{array}{l}.77 \\
.04 \\
.61\end{array}$ & .57 \\
\hline $\begin{array}{l}\text { 3. Control medio de super- } \\
\text { visores de otras unidades } \\
\text { a. Sobre las operaciones } \\
\text { de la unidad } \\
\text { b. Sobre todo el hospital } \\
\text { c. Sobre eventos y cir- } \\
\text { cunstancias externas }\end{array}$ & $\begin{array}{l}.70 \\
-\end{array}$ & $\begin{array}{l}.82 \\
.35 \\
-\end{array}$ & $\begin{array}{l}.72 \\
.21 \\
.45 \\
-\end{array}$ & .60 & $\begin{array}{l}\text { 4. Control medio del direc- } \\
\text { tor médico } \\
\text { a. Sobre las operaciones } \\
\text { de la unidad } \\
\text { b. Sobre todo el hospital } \\
\text { c. Sobre eventos y cir- } \\
\text { cunstancias externas }\end{array}$ & $\begin{array}{l}.79 \\
-\end{array}$ & $\begin{array}{l}.80 \\
.40 \\
-\end{array}$ & $\begin{array}{l}.78 \\
.14 \\
.49\end{array}$ & .59 \\
\hline $\begin{array}{l}\text { 5. Control medio del ge- } \\
\text { rente } \\
\text { a. Sobre las operaciones } \\
\text { de la unidad } \\
\text { b. Sobre todo el hospital } \\
\text { c. Sobre eventos y cir- } \\
\text { cunstancias externas }\end{array}$ & .83 & $\begin{array}{l}.74 \\
.42 \\
-\end{array}$ & $\begin{array}{l}.70 \\
.26 \\
.54 \\
-\end{array}$ & .56 & $\begin{array}{l}\text { 6. Control medio de la je- } \\
\text { fa de personal } \\
\text { a. Sobre las operaciones } \\
\text { de la unidad } \\
\text { b. Sobre todo el hospital } \\
\text { c. Sobre evenros y rir. } \\
\text { cunstancias externas }\end{array}$ & $\begin{array}{l}.80 \\
-\end{array}$ & $\begin{array}{l}.83 \\
.48 \\
-\end{array}$ & $\begin{array}{l}.82 \\
.44 \\
.59\end{array}$ & .73 \\
\hline $\begin{array}{l}\text { 7. Control medio del comi- } \\
\text { té de empresa } \\
\text { a. Sobre las operaciones } \\
\text { de la unidad } \\
\text { b. Sobre todo el hospital } \\
\text { c. Sobre eventos y cir- } \\
\text { cunstancias externas }\end{array}$ & .65 & $\begin{array}{l}.80 \\
.35 \\
-\end{array}$ & $\begin{array}{l}.76 \\
.21 \\
.42\end{array}$ & .58 & $\begin{array}{l}\text { 8. Control medio de perso- } \\
\text { nas ajenas al hospital } \\
\text { a. Sobre las operaciones } \\
\text { de la unidad } \\
\text { b. Sobre todo el hospital } \\
\text { c. Sobre eventos y cir- } \\
\text { cunstancias externas }\end{array}$ & $\begin{array}{l}.71 \\
-\end{array}$ & $\begin{array}{l}.88 \\
.50 \\
-\end{array}$ & $\begin{array}{l}.87 \\
.42 \\
.65\end{array}$ & .76 \\
\hline
\end{tabular}

Los valores calculados para estos índices de influencia global se encuentran en la tabla I y en la figura 2 el correspondiente gráfico de control. La pendiente de este gráfico arroja un valor de $(\mathrm{P}=-.32)$. Aunque la diferencia absoluta entre el máximo (4.27) correspondiente al gerente y el mínimo (1.97) correspondiente a los miembros es de más de dos puntos, el valor relativamente bajo de la pendiente se explica por las diferencias mínimas de control total que se dan entre determinados niveles.

Aspectos a resaltar son el escaso control percibido de los miembros, que prácticamente detentan la misma cantidad de control global que las personas ajenas al hospital, y que el único nivel al que se percibe con «bastante» 0 «mucho» control es el correspondiente al gerente en tanto que el director está ligeramente por encima del punto correspondiente a salgos de control. 


\section{c) Distribución del control percibido a través de las unidades}

Siguiendo con los resultados descriptivos, la tabla III muestra la distribución del control global a través de todas las unidades. Estos datos se encuentran representados en la figura 3. La inspección visual nos señala que en líneas generales no existen grandes diferencias en la estructura de control percibida por cada unidad para cada grupo o posición. La gráfica muestra una estratificación que se corresponde casi exactamente con la esperada en función de la localización en el organigrama de cada grupo o posición, dándose algunas excepciones en determinadas unidades. Una de estas excepciones es el alto control que atribuye la unidad de administración a su supervisor. La razón de este dato se explica en la medida en que el supervisor de esta unidad es el gerente. Las unidades que perciben con menor control relativo a los cargos administrativos - gerente, jefa de personal y director- son el laboratorio, la unidad de especialidades, la de médicos de salas y la de médicos de consultas externas, esto es, las de mayor componente profesional.

TABLA III

Estadísticos básicos del control global de cada posición desglosado por unidades

\begin{tabular}{|c|c|c|c|c|c|c|c|c|c|c|c|c|c|c|c|c|}
\hline \multirow{3}{*}{ UNIDAD } & \multicolumn{16}{|c|}{ POSICIONES DE LA JERARQUIA } \\
\hline & \multicolumn{2}{|c|}{ Miembros } & \multicolumn{2}{|c|}{ Superv. } & \multicolumn{2}{|c|}{ Otros. Sup. } & \multicolumn{2}{|c|}{ Dtor. Méd. } & \multicolumn{2}{|c|}{ Gerente } & \multicolumn{2}{|c|}{ Jefa Per. } & \multicolumn{2}{|c|}{ Cte. Emp. } & \multicolumn{2}{|c|}{ P. Ajenas } \\
\hline & $\overline{\mathbf{X}}$ & DT & $\overline{\mathbf{X}}$ & DT & $\overline{\mathbf{X}}$ & DT & $\overline{\mathbf{X}}$ & DT & $\overline{\mathbf{X}}$ & DT & $\overline{\mathbf{x}}$ & DT & $\overline{\mathbf{X}}$ & DT & $\overline{\mathbf{X}}$ & $\mathbf{D r}$ \\
\hline LIMP & 1.56 & .70 & 3.06 & .64 & 1.96 & 1.01 & 3.06 & .90 & 4.56 & .52 & 3.83 & .70 & 2.70 & .76 & 1.66 & .70 \\
\hline TELE & 1.66 & .33 & 2.66 & .66 & 2.11 & 1.01 & 3.88 & .38 & 4.88 & .19 & 3.33 & .57 & 2.00 & .57 & 2.22 & 2.11 \\
\hline LENCE & 2.20 & .55 & 2.65 & .68 & 1.86 & .50 & 3.06 & .68 & 3.73 & .59 & 3.06 & .92 & 2.06 & .43 & 2.00 & .62 \\
\hline RECEP & 1.66 & .72 & 1.83 & .57 & 2.58 & 1.10 & 3.66 & .81 & 5.00 & .00 & 2.94 & .43 & 2.33 & .72 & 2.00 & .94 \\
\hline COCIN & 2.06 & .54 & 2.80 & .60 & 1.73 & 1.60 & 3.29 & .38 & 4.33 & .52 & 2.93 & .36 & 2.13 & .73 & 2.93 & .76 \\
\hline ADMI & 1.90 & .63 & 4.24 & .95 & 2.24 & .85 & 3.45 & .94 & 4.63 & .45 & 2.36 & .81 & 1.87 & .68 & 1.72 & .85 \\
\hline $\mathrm{CIR}$ & 2.26 & 1.09 & 2.70 & 1.13 & 1.99 & 1.12 & 4.00 & .81 & 4.66 & .58 & 3.24 & 1.15 & 2.23 & .81 & 1.77 & 1.16 \\
\hline URGN-A & 1.86 & 1.07 & 2.47 & 1.07 & 1.87 & 0.71 & 3.36 & .87 & 5.00 & .00 & 3.43 & .62 & 2.20 & .65 & 3.59 & 1.20 \\
\hline URGN-B & 2.38 & 1.42 & 2.68 & 1.21 & $\mid 1.62$ & 0.49 & 4.06 & 1.11 & 4.20 & .79 & 3.63 & .85 & 2.26 & .98 & 2.05 & .85 \\
\hline ESC-A & 1.44 & .80 & 2.66 & .89 & 1.72 & .61 & 3.94 & 1.27 & 4.33 & .63 & 4.22 & .88 & 3.00 & .55 & 1.22 & .27 \\
\hline ESC-B & 2.38 & 1.43 & 1.83 & .27 & 1.72 & .80 & 2.50 & .81 & 4.33 & .76 & 2.77 & .45 & 3.00 & .76 & 2.88 & 1.47 \\
\hline INFE & 2.20 & 1.01 & 3.00 & .62 & 2.20 & .90 & 4.26 & .27 & 4.66 & .40 & 3.80 & 1.04 & 2.93 & .14 & 2.06 & 1.38 \\
\hline LACT & 2.00 & .47 & 2.66 & .66 & 1.41 & .63 & 3.80 & .65 & 4.16 & .88 & 2.94 & .94 & 2.08 & .73 & 1.15 & .33 \\
\hline LABO & 2.00 & .42 & 2.83 & .28 & 2.25 & .69 & 3.04 & .43 & 3.29 & .64 & 2.02 & .47 & 1.83 & .86 & 1.20 & .40 \\
\hline ESPE & 2.06 & .57 & 2.57 & 1.23 & 1.83 & .67 & 3.23 & .98 & 3.70 & .61 & 2.61 & .84 & 1.69 & .73 & 1.61 & .57 \\
\hline SALA & 2.12 & 1.00 & 2.94 & 1.11 & 2.18 & .81 & 3.61 & 1.35 & 3.42 & .93 & 2.61 & .57 & 1.94 & .55 & 2.70 & 1.27 \\
\hline URGE & 1.86 & .76 & 3.46 & .58 & 2.12 & .96 & 3.61 & .52 & 4.14 & .37 & 2.04 & .78 & 2.04 & .65 & 1.66 & .94 \\
\hline
\end{tabular}

LEYENDA: LIMP $=U$. Limpieza: TELE $=U$. Teléfonos; LENCE = U. Lencería; RECEP $=$ U. Recepción; COCIN = U. Cocina; ADMIN = U. Administración; CIR = U. Cirugía; URGAN A y B $=$ U. Urgencias Nocturnas; ESCA y B = Salas de Escolares A y B; INFE = Sala de Infecciosos; LACT = Sala de Lactantes; $\mathrm{LABO}=$ Laboratorio; ESPE $=$ Médicos especialistas; SALA = Médicos pediatras; URGE = Médicos Servicios de Urgencias. 
FIGURA 3

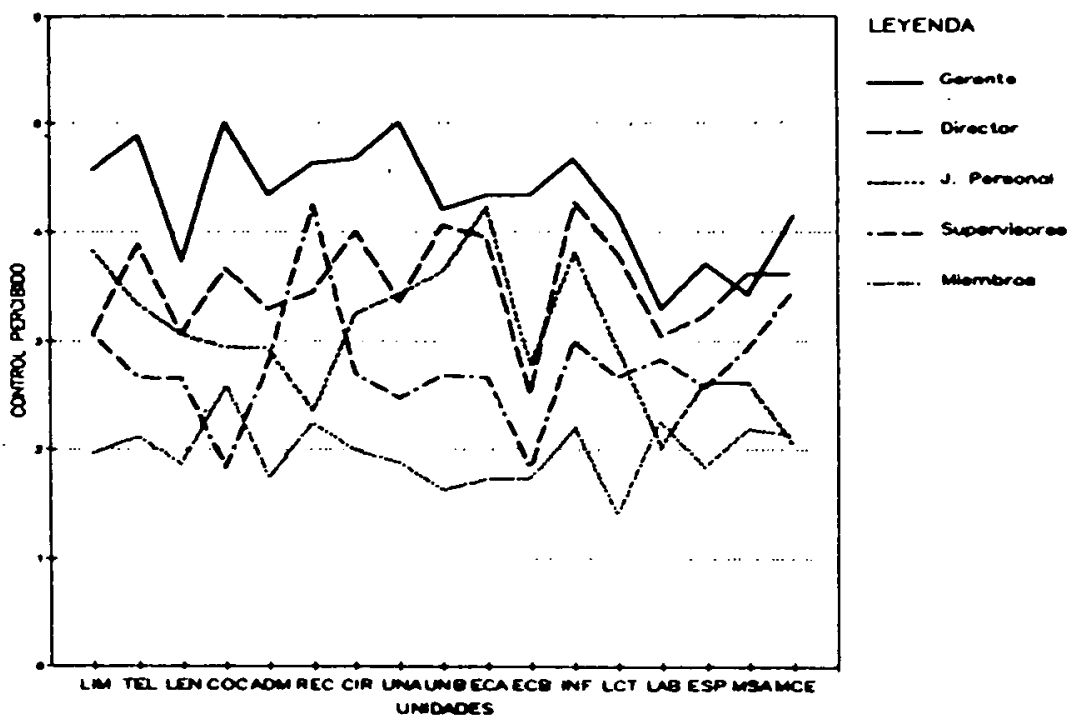

Control percibido de las distintas posiciones para cada unidad.

d) Distribución del control percibido por niveles jerárquicos

La figura 4 muestra los gráficos correspondentes a los tres niveles colectivos: empleados de primera línea (nivel 0 ), supervisores de primera línea (nivel 1) y jefes de de servicio (nivel 2). En la gráfica se aprecia que aunque varía la cantidad de control percibido para cada uno de los grupos o posiciones por los miem-

FILUUKA 4

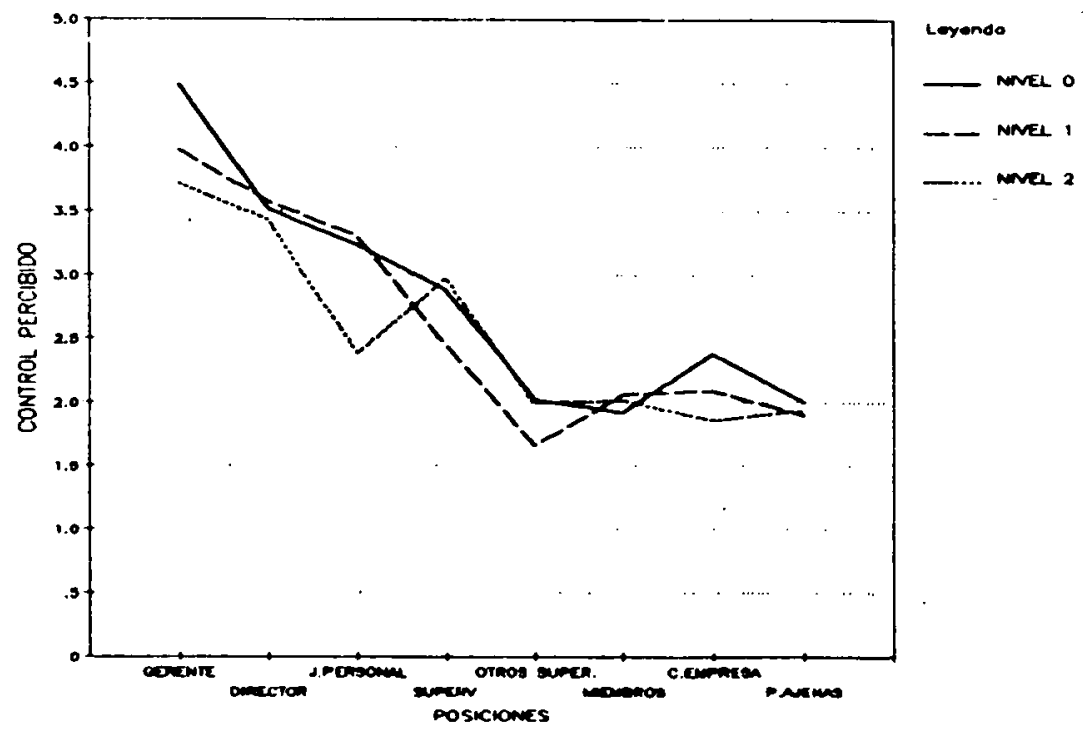

Control percibido global de las distintas posiciones por niveles. 
bros de cada nivel, la forma del gráfico indica que la distribución relativa del control entre los distintos grupos o posiciones permanece constante. La única excepción ocurre para la jefa de personal en el nivel 2 que corresponde a los médicos y jefes de servicios. La cantidad de control atribuido por los niveles 1 (en su mayor parte Enfermeras) y el 2 (Médicos y Jefes de Servicios) a los cargos administrativos disminuye apreciablemente, aunque esto no supone un incremento apreciable en el control de los miembros pertenecientes a dichos niveles.

\section{e) Relaciōn entre localizaciones estructurales y estructura percibida de control}

Con el objeto de comprobar el efecto de las distintas localizaciones estructurales y funcionales de los miembros sobre la estructura percibida de control llevamos a cabo tres análisis de la varianza categorizando a los sujetos por unidades, niveles y puestos. Para el caso de los niveles se eliminaron las tres posiciones más altas por ser unipersonales. Los puestos fueron agrupados en 7 categorías tomando como criterio de pertenencia la similitud de actividades y especificaciones de los mismos. Las categorías establecidas fueron las siguientes: Servicios (25 ocupantes), Administración (10 ocupantes), Auxiliares Clínicos (37 ocupantes), Enfermeras (12 ocupantes), Médicos Generales ( 7 ocupantes), Médicos Pediatras ( 8 ocupantes) y Médicos de Especialidades ( 9 ocupantes). En todos los casos se controló el requisito de homogeneidad de la varianza mediante la prueba $« C »$ de Cochrans, calculándose el coeficiente ETA $^{2}$ con el fin de estimar el porcentaje de varianza del control percibido de cada grupo o posición explicado por la pertenencia a las distintas localizaciones estructurales.

Los resultados se presentan en las tablas IV, V y VI. Estos muestras que para las unidades se encuentran diferencias significativas en el control percibido de casi todos los grupos o posiciones, excepto para los miembros y otros supervisores. Las diferencias significativas alcanzan un nivel de al menos $\mathrm{p}<.01$ excepto para el director que es de $\mathrm{p}<.058$ con una $\left(\mathrm{F}_{16,93}=1.71\right)$. Los valores de $\mathrm{E}^{2}$ son bastante altos para casi todos los grupos, especialmente para la Jefa de Personal $\left(\mathrm{ETA}^{2}=.62\right)$, y el gerente $\left(\mathrm{ETA}^{2}=.44\right)$.

TABLA IV

Valores de "F", ETA cuadrado y nivel de significación para la localización por unidades

\begin{tabular}{|l|c|c|c|}
\hline \multicolumn{1}{|c|}{ Nivel } & F & ETA cuadrado & P \\
\hline Gerente & 4.71 & .44 & .000 \\
Director & 1.71 & .22 & .058 \\
Jefa Personal & 4.26 & .62 & .000 \\
Supervisores & 3.00 & .34 & .000 \\
Otros Supervisores & 0.58 & .09 & .891 \\
Miembros & 0.65 & .10 & .834 \\
Comité Empresa & 2.22 & .27 & .009 \\
Personas Ajenas & 2.80 & .33 & .001 \\
Pendiente & 2.77 & .32 & .002 \\
\hline
\end{tabular}


TABLA V

Valores de .F”, ETA cuadrado y nivel de significación para la localizaciōn por niveles

\begin{tabular}{|l|c|c|c|}
\hline \multicolumn{1}{|c|}{ Nivel } & F & ETA cuadrado & P \\
\hline Gerente & 13.61 & .20 & .000 \\
Director & 0.08 & .00 & .906 \\
Jeŕa Personal & 8.81 & .14 & .003 \\
Supervisores & 1.06 & .01 & .349 \\
Otros Supervisores & 1.92 & .07 & .398 \\
Miembros & 1.19 & .00 & .826 \\
Comité Empresa & 4.58 & .07 & .001 \\
Personas Ajenas & 0.06 & .00 & .093 \\
Pendiente & 3.59 & .06 & .003 \\
\hline
\end{tabular}

TABLA VI

Valores de "F", ETA cuadrado y nivel de significación para la localización por puestos

\begin{tabular}{|l|c|c|c|}
\hline \multicolumn{1}{|c|}{ Nivel } & F & ETA cuadrado & P \\
\hline Gerente & 8.35 & .36 & .000 \\
Director & 0.87 & .00 & .515 \\
Jefa Personal & 5.99 & .30 & .000 \\
Supervisores & 2.51 & .13 & .026 \\
Otros Sunperyisnres & 0.90 & .06 &.$j 1 i$ \\
Miembros & $0.42^{-}$ & .03 & .001 \\
Comité Empresa & 2.66 & .15 & .001 \\
Personas Ajenas & 0.50 & .06 & .800 \\
Pendiente & 2.47 & .16 & .017 \\
\hline
\end{tabular}

Para los niveles, los efectos significativos se reducen a sólo tres de los grupos: el gerente $\left(\mathrm{F}_{2,107}=13.61 ; \mathrm{p}<.000\right)$; la jefa de personal $\left(\mathrm{F}_{2.107}=8.81\right.$; $\mathrm{p}<.003)$; y el comité de empresa $\left(\mathrm{F}_{2,107}=4.58 ; \mathrm{p}<.001\right)$ y para la pendiente $(\mathrm{P})\left(\mathrm{F}_{2,106}=3.59 ; \mathrm{p}<.003\right)$. En este caso la varianza explicada es bastante inferior a la de las unidades destacando solamente la del control percibido del gerente $\left(\mathrm{ETA}^{2}=.20\right)$.

La pertenencia a las categorías de puestos discrimina significativamente en el control percibido de cuatro de los grupos o posiciones: el gerente $\left(\mathrm{F}_{7,104}=8.35 ; \mathrm{p}<.000\right)$; La jefa de personal $\left(\mathrm{F}_{7,104}=5.99 ; \mathrm{p}<.00\right)$; los supervisores $\left(\mathrm{F}_{7,104}=2.51 ; \mathrm{p}<.026\right)$; el comité de empresa $\left(\mathrm{F}_{7,104}=2.66\right.$; $\mathrm{p}<.001)$; $y$ la pendiente $\left(\mathrm{F}_{7,102}=2.47 ; \mathrm{p}<.017\right)$. La varianza explicada es apreciable para el caso del gerente $\left(\mathrm{ETA}^{2}=.36\right)$ y la jefa de personal $\left(\mathrm{ETA}^{2}=.30\right)$, no así para el resto de los grupos. 
f) Correlatos del control percibido con el contexto, diseño y resultados de los puestos y unidades

La tabla VIII presenta los coeficientes de correlación de Pearson entre los índices de control percibido de los distintos grupos y posiciones y los índices de contexto, diseño y resultados de los puestos. Como síntesis podemos resaltar la correlación negativa que presenta el nivel de experto con el control percibido del Gerente $(\mathrm{r}=-.30 ; \mathrm{p}<.01)$, de la jefa de personal $(\mathrm{r}=-.39 ; \mathrm{p}<.01)$ y del comité de empresa $(r=-34)$. La expectativas de sanciones correlacionan positivamente con el control percibido del gerente $(\mathrm{r}=.24 ; \mathrm{p}<.01)$, en tanto que las de gratificaciones lo hacen negativamente con éste $(r=-.21 ; p<.01)$ y positivamente con el control percibido del director $(r=.20 ; \mathrm{p}<.05)$.

\section{TABLA VII}

Correlaciones de los indices de control global con los indices de contexto, diseño y resultados del puesto

\begin{tabular}{|c|c|c|c|c|c|c|c|c|c|}
\hline \multirow{2}{*}{$\begin{array}{c}\text { INDICES } \\
\text { DEL } \\
\text { PUESTO }\end{array}$} & \multicolumn{9}{|c|}{ INDICES DE CONTROL } \\
\hline & $\begin{array}{l}\text { Control } \\
\text { Gerente }\end{array}$ & $\begin{array}{l}\text { Control } \\
\text { Director }\end{array}$ & $\begin{array}{l}\text { Control } \\
\text { J. Pers. }\end{array}$ & $\begin{array}{l}\text { Control } \\
\text { Supervs. }\end{array}$ & $\begin{array}{l}\text { Control } \\
\text { S. Superv. }\end{array}$ & $\begin{array}{c}\text { Control } \\
\text { Miembros }\end{array}$ & $\begin{array}{l}\text { Control } \\
\text { C. Emp. }\end{array}$ & $\begin{array}{l}\text { Control } \\
\text { P. Ajen. }\end{array}$ & Pendiente \\
\hline CONTEXTO & & & & & & & & & \\
\hline Dif. de la Tarea. &. .13 & .02 & .05 & .00 & .08 & .05 & .03 & .00 & .08 \\
\hline Var. de la Tarea. & .00 & .09 & $.22^{* *}$ & .05 & -.09 & .18 & .02 &. .15 & -.14 \\
\hline Int. Nec. Creci. & .01 & .05 & -.08 & -.14 & .06 & .04 & -.02 & .11 & .11 \\
\hline DISEÑO & & & & & & & & & \\
\hline Estandarización & .09 & .12 & .06 & .07 & -.01 & .06 & .06 & .01 & .07 \\
\hline Niv. Experto. & $.30^{* * *}$ & .09 & $-.39^{* * *}$ & .00 & .05 & .03 & $.34^{* * *}$ & .03 & .15 \\
\hline Autoridad. & $-27^{* * *}$ & .08 &. $.18^{*}$ & .17 & .03 & .18 & .10 &. .14 & .03 \\
\hline Presión. & .06 & .09 & .13 & $.29^{* * *}$ & .02 &. .06 & .11 & .02 & .06 \\
\hline Respons. Ex. & .02 & $.24^{* *}$ & -.12 & .13 &. .15 & .12 & .00 & .01 & .03 \\
\hline Respons. Int. & -.08 & -.07 & .08 & -.12 & .07 &. .11 & .02 & .05 & .10 \\
\hline Ret. del Puesto. & .10 & .10 & -.19 & .11 & .04 & -.00 & .19 & .06 & .13 \\
\hline Ret. de Compa. & .03 & .15 & .12 & -.09 & .09 & .02 & .02 & .09 &. .11 \\
\hline Ret. Superv. & .09 & .14 & .16 & .14 & $.20^{* *}$ & .07 & .09 & .03 & .01 \\
\hline Expect. Castigo. & $.24^{* * *}$ & .04 & .17 & .15 & -.11 & .06 & .04 & .16 & .01 \\
\hline Expect. Recomp. & $.21^{* * *}$ & $.20^{* *}$ & .06 & .18 & .12 & .25 & .12 & .00 & .12 \\
\hline RESULTADOS & & & & & & & & & \\
\hline Esfuerzo & $.40^{* * *}$ & $.25^{* * *}$ & .16 & .13 & .01 & $.0 \mathrm{i}$ & .08 & .11 & $.35 * * *$ \\
\hline Mot. Interna. & & & .19 & .04 & .17 & .07 & .11 & .11 & .02 \\
\hline Sat. Trabajo & $.22^{* * *}$ & $.30^{* * *}$ & $.22^{* *}$ & .12 & .03 & .21 & .08 & ..11 & $.26^{* * *}$ \\
\hline Sat. Superv. & & $.20^{* *}$ & .16 & .05 & -.19 & .12 & .10 & .03 & -.13 \\
\hline Sat. Salario. & $.30^{* * *}$ & .01 & .07 & .06 & .14 & .05 & .12 & .06 & -.14 \\
\hline Sat. Compañe. & .04 & .10 & .01 & .02 & .18 & .15 & .02 & .05 & \\
\hline Sat. Progres. & .08 & .19 & .10 & .07 & -.07 & $.28^{* * *}$ & $.26^{* * *}$ & .05 & .00 \\
\hline Sat. Carrera. & $\cdot .14$ & .13 & .10 & -.03 & -.07 & .06 & $.20^{* *}$ & .08 & .00 \\
\hline Sat. General & .09 & $.26^{* * *}$ & $.22^{* *}$ & .09 & -.16 & $.20^{* *}$ & $.24 * * *$ & .05 & .00 \\
\hline
\end{tabular}

$110<\mathrm{N}<113 ;$ gl. $=97 i^{*}=\mathrm{p}<.10 ;{ }^{* *}=\mathrm{p}<05 ;{ }^{* * *}=\mathrm{p}<.01$.

Las correlaciones significativas con los índices de resultados son todas positivas excepto para la satisfacción con el salario en el caso del control percibido del gerente $(\mathrm{r}=-.30 ; \mathrm{p}<.01)$. El indice de satisfacción general correlaciona positivamente con el control percibido de miembros $(\mathrm{r}=.20 ; \mathrm{p}<.05)$ y del comité de empresa $(r=.24 ; \mathrm{p}<.01)$. 
Dado que. tanto el nivel experto, como la localización y la antigüedad de los miembros, podría estar afectando los resultados descritos anteriormente, llevamos a cabo un análisis de correlación parcial controlando los efectos de dichas variables.

La tabla VIII muestra estos resultados. Se aprecia efectivamente el efecto modulador de estas variables que se manifiesta en un incremento general en las correlaciones del control percibido de los miembros con las variables de satisfacción.

TABLA VIII

Correlaciones parciales del control percibido con los indices de resultados del puesto controladas para el nivel de experto, nivel jerárquico y antigüedad

\begin{tabular}{|c|c|c|c|c|c|c|c|c|c|}
\hline \multirow{2}{*}{ RESULTADOS } & \multicolumn{9}{|c|}{ INDICES DE CONTROL } \\
\hline & $\begin{array}{l}\text { Control } \\
\text { Gerente }\end{array}$ & $\begin{array}{l}\text { Control } \\
\text { Director }\end{array}$ & $\begin{array}{l}\text { Control } \\
\text { J. Pers. }\end{array}$ & $\begin{array}{c}\text { Control } \\
\text { Superss. }\end{array}$ & $\begin{array}{l}\text { Control } \\
\text { S. Superr. }\end{array}$ & $\begin{array}{c}\text { Control } \\
\text { Miembros }\end{array}$ & $\begin{array}{l}\text { Control } \\
\text { C. Emp. }\end{array}$ & $\begin{array}{l}\text { Control } \\
\text { P. Ajen. }\end{array}$ & Pendiente \\
\hline Sat. con trabajo & $.21^{* *}$ & $.33^{* * *}$ & .12 & .10 & $.03^{\circ}$ & $.26 * * *$ & .04 & .12 & $.24^{* * *}$ \\
\hline Sat. con Superii. & .06 & $.25^{* * *}$ & $.23 * *$ & .07 & .19 & .13 & $.18^{*}$ & .04 & .00 \\
\hline Sat. con Salario. & .08 & .00 & .15 & .05 & .07 & .07 & $.25 * * *$ & .04 & .08 \\
\hline Sat. con Compañe. & .00 & .10 & .06 & .01 & $.17^{*}$ & $.15^{*}$ & .02 & .07 & .07 \\
\hline Sat. con Promoción. & .00 & $.21^{* *}$ & .11 & .10 & .03 & $.30^{* * *}$ & $.31^{* * *}$ & .09 & .08 \\
\hline Sat. con Op. Fut. &. .16 & .15 & .15 & .02 & .01 & .06 & $.27 * * *$ & .12 & .02 \\
\hline Sat. General. & .02 & $.30^{* * *}$ & $.10^{* *}$ & .10 & .15 & $.24 * * *$ & $.28^{* * *}$ & -.11 & .09 \\
\hline Effuerzo. & $.36 * * *$ & $.25^{* * *}$ & .00 & $.17^{*}$ & .00 & .03 & .05 & -.13 & $.33^{* * * 1}$ \\
\hline
\end{tabular}

$110<N<113:$ gl. $=97^{*}=p<.10:{ }^{* *}=p<.05:^{* * *}=p<.01$

Las correlaciones del control percibido del director sufren también un incremento apreciable. Así se incrementa la correlación con la satisfacción en el trabajo $(\mathrm{r}=.33 ; \mathrm{p}<.01)$; con la supervisión $(\mathrm{r}=.24 ; \mathrm{p}<.01)$; con la promoción $(\mathrm{r}=.22 ; \mathrm{p}<.01)$, que antes no era significativa y por ende, con la satisfacción general $(\mathrm{r}=.30 ; \mathrm{p}<.01)$.

Las correlaciones de los índices control entre sí y con los índices de contexto, diseño y resultado de las unidades, agregados a este nivel, se pueden ver en la tabla IX. Puesto que al agregar las respuestas de los individuos al nivel de las unidades se redujo a veinte el número de casos que entran en el análisis, el coeficiente calculado fue el de rangos de Spearman. Entre estos resultados podemos destacar que el nivel de experto de las unidades correlaciona negativamente con el control percibido del gerente $(\tau=-.60 ; \mathrm{p}<.01)$, y de la jefa de personal $(\tau=-.48 ; \mathrm{p}<.05)$.

El control de los miembros correlaciona negativamente, con el conflicto entre los miembros $(\tau=-.39 ; \mathrm{p}<.05)$; también correlaciona positivamente con la eficiencia percibida de las unidades $(\tau=.41 ; \mathrm{p}<.05)$.

El control del supervisor correlaciona positivamente con el número de objetivos cubiertos por la unidad $(\tau=.56 ; \mathrm{p}<.01)$.

El control del gerente correlaciona positivamente con el del director médico $(\tau=.37 ; \mathrm{p}<.05)$, el de la jefa de personal $(\tau=.52 ; \mathrm{p}<.05)$ y con el del comité de empresa $(\tau=.40 ; \mathrm{p}<.05)$. 


\section{4}

TABLA IX

Correlaciones de los indices de control global con los indices de contexto, diseño y resultados de la unidad

\begin{tabular}{|c|c|c|c|c|c|c|c|c|}
\hline \multirow{2}{*}{$\begin{array}{c}\text { INDICES } \\
\text { DE LA } \\
\text { UNIDAD }\end{array}$} & \multicolumn{8}{|c|}{ INDICES DE CONTROL } \\
\hline & $\begin{array}{l}\text { Control } \\
\text { Gerente }\end{array}$ & $\begin{array}{l}\text { Control } \\
\text { Director }\end{array}$ & $\begin{array}{l}\text { Control } \\
\text { J. Pers. }\end{array}$ & $\begin{array}{l}\text { Control } \\
\text { Superrs. }\end{array}$ & $\begin{array}{l}\text { Control } \\
\text { S. Superv. }\end{array}$ & $\begin{array}{l}\text { Control } \\
\text { Miembros }\end{array}$ & $\begin{array}{l}\text { Control } \\
\text { C. Emp. }\end{array}$ & $\begin{array}{l}\text { Control } \\
\text { P. Ajen. }\end{array}$ \\
\hline $\begin{array}{l}\text { DISEÑO } \\
\text { Intercambiabilidad de roles }\end{array}$ & 14 & 08 & 23 & -12 & .09 & 03 & 30 & -01 \\
\hline $\begin{array}{l}\text { Estandarización } \\
\text { Autoridad: }\end{array}$ & .05 & .01 & .09 & .03 & .01 & .03 & .21 & .08 \\
\hline Supervisor & .05 & .23 & .02 & .41 & .08 & .14 & .11 &. .22 \\
\hline Empleados & -.13 & .00 & .05 & -.02 & .08 & .12 & .03 &. .11 \\
\hline Colegiada & .09 & .25 & .04 & .11 & .06 & .16 & .23 & -.13 \\
\hline Externa & .32 & .15 & .12 & .14 & .03 & .00 & .01 & .23 \\
\hline Niv. Experto & .60 & .04 & .48 & .14 & .02 & .26 &. .27 & .26 \\
\hline DEPENDENCIA & & & & & & & & \\
\hline Del Supervisor & .16 & .28 & .23 & .22 & .09 & .05 & .23 & -.07 \\
\hline De los Miembros & .10 & .05 & .08 &. .10 & .10 & .23 & .07 & .15 \\
\hline De otras Unidades & .12 & -.01 & .08 & .28 & .04 & .01 & .08 & .23 \\
\hline COMUNICACION & & & & & & & & \\
\hline Escrita & .19 & $\cdot .04$ &. .19 & -.12 & .04 & .05 & -.11 & .08 \\
\hline Personal & -.08 & .05 & .15 & .00 & .03 & .04 & .01 & .08 \\
\hline Gupal & -.57 & .33 &. .37 & .10 & .10 & .12 & .37 & .21 \\
\hline CONFLICTO & & & & & & & & \\
\hline Con supervisor & .15 & .02 & .11 & .11 & .69 & .35 & .12 & .03 \\
\hline Con compañeros & .06 & .03 & -.07 & .07 & . 06 & .39 & .07 & .08 \\
\hline Con otras Unidades & .05 &. .13 & -.20 &. .12 & $.5 i$ & .08 &. .25 & .08 \\
\hline RENDIMIENTO & & & & & & & & \\
\hline Eficiencia & .33 & .30 & .04 & .19 & .06 & .41 & .05 & -.14 \\
\hline Objetivos Cubiertos & -.18 & -.17 & -.18 & .56 & .02 & .04 & .16 & .03 \\
\hline Moral & .12 & .07 & .03 & .18 & .33 & .03 & .08 & .11 \\
\hline Rendimiento global & .07 & .11 & -.18 & .01 & .08 & .11 & .06 &. .11 \\
\hline
\end{tabular}

Todos los coeficientes $>.40$ son significativos a un nivel de al menos $\mathrm{p}>.05$.

\section{DISCUSION}

En este trabajo hemos examinado la estructura percibida de control de una organización hospitalaria de tamaño relativamente pequeño. Los resultados con el gráfico de control muestran que la estructura percibida de control e influencia se ajusta a los patrones de una configuración jerárquica clásica, que da como resultado un gráfico de pendiente negativa. La distribución del control por niveles es aproximadamente la misma, aunque varíe la cantidad total de control percibido para cada uno de los grupos, pero que aunque esta cantidad varía, la distribución relativa de control percibido entre los distintos grupos permanece constante. Esto confirma los resultados obtenidos por otros autores (Bouchar, 1976; Van de Ven y Ferry, 1980), en el sentido de que la posición vertical y horizontal que ocupa el informante en la organización afecta a sus juicios sobre el comportamiento organizacional, y por tanto no son generalizables. La conclusión que se extrae de esto y que tiene evidentes repercusiones metodológicas es que para tener una idea ajustada de los fenómenos organizacionales es necesario extraer la información a partir de todos los niveles y posiciones. 
El diseño de la estructura de control es por tanto estrictamente jerárquico. En ésta domina el papel del gerente cuya influencia percibida es superior al del resto de las posiciones en todas las áreas, incluso en la de las actividades internas de las unidades. Las distintas distribuciones del control — por niveles, áreas de influencia, y unidades - confirman una estructura altamente centralizada, en la que los miembros tienen un escaso control para tomar decisiones en las diferentes áreas.

La varianza en la cantidad de influencia percibida se explica en mayor medida por la localización de los miembros en las unidades que su pertenencia a los distintos niveles jerárquicos o a las categorías de puestos. Esto es consistente con los resultados obtenidos entre otros por Markham (1984), que apuntan a que la estructura de control de las distintas unidades es diferente, variando los estilos y procedimientos de supervisión, comunicación e incentivación. Otra posible interpretación, compatible con la anterior, es que las personas pertenecientes a las distintas unidades no concuerdan en la estructura percibida de autoridad porque disponen de diferente información, distinta experiencia cotidiana con las distintas posiciones evaluadas e incluso distintos antecedentes en el hospital. Sin embargo algo en lo que son consistentes todos los miembros, a pesar de las diferencias comentadas anteriormente es en el alto grado de centralización de la estructura de control en la figura del gerente.

Las correlaciones de los índices de control con los índices de contexto, diseño y resultados de los puestos y unidades ayudan a clarificar la naturaleza de las relaciones de poder y su impacto sobre diversos procesos. Así, respecto de las unidades el control del gerente se asocia a la autoridad externa, la baja participación grupal y la conflictividad con el supervisor. La del director se asocia a la autoridad del supervisor y a la distribución de influencia a través de métodos grupales, y el control de la jefa de personal a la intercambiabilidad de roles (se explica en la medida que supervisa directamente las unidades de limpieza y lavandería) y a la conflictividad con otras unidades.

Kespecto de los puestos el perfil del gerente estaría asociado a una baja percepción de su autoridad por parte de las personas con mayor nivel de experto, a una baja expectativa de gratificaciones y alta expectativa de sanciones por parte de los ocupantes de los puestos; asimismo, se asocia a una baja satisfacción con el salario y un alto esfuerzo en el trabajo.

Estos resultados confirman el diseño de burocracia profesional característico de este tipo de organizaciones. Aparecen claramente dibujadas las dos líneas de control: la administrativa, altamente centralizada en la figura del gerente que confiere a los miembros una escasa participación en la toma de decisiones y que está asociada a procedimientos de control basados en el individuo y el recurso a la sanción. La segunda línea de control, la profesional, aparece más descentralizada, y el control percibido de su cabeza, el director médico, se asocia a procedimientos grupales de toma de decisión.

También los resultados parecen apoyar la naturaleza no absoluta del reparto del poder. Se puede apreciar en los resultados que un aumento en la influencia de uno de los grupos o posiciones en una determinada área no parece llevar parejo un decremento lineal de otros. Esto se puede apreciar en las correlaciones positivas que arrojan la influencia del gerente y el director médico o la del gerente y el comité de empresa. Este último dato es especialmente significativo en la medida; estas dos figuras representan intereses en muchas ocasiones contrapuestos y se ven sometidos a roces y conflictos casi permanentes. Esta conclu- 
sión se ve también corroborada por el hecho de que a pesar de que en los niveles más profesionalizados se aprecia una disminución en el control percibido de la línea administrativa y en especial del gerente, esto no va acompañado por un incremento en el control de los miembros de dichos niveles.

En resumen, creemos que los datos y resultados obtenidos sobre la estructura de control mediante el gráfico de Tannenbaum aportan una información interesante y altamente fiable para la explicación de la estructura percibida de poder de esta organización. Al margen de su interés puramente académico, estos resultados permiten tomar decisiones y sobre todo retroalimentar a la organización dándole una información cuantitativa y gráfica de esta estructura aplicable al cambio y desarrollo planificado de la misma. Asimismo, el método arroja una buena validez convergente y discriminante para diferenciar entre los distintos grupos y posiciones tanto verticales como horizontales y confirma la importancia que tiene, en orden al desarrollo y cambio planificado de estas organizaciones, la consideración del carácter altamente profesionalizado de los sectores dedicados directamente a la producción de los servicios (Filley et al., 1976).

\section{Referencias}

BLAU, P.M. y SCHOENHERR, R.A. (1971). The Structure of Organizations. Nueva York. Basic Books.

BlaU. P.M. y ScOTT, W.R. (1962). Formal Organizations: A Comparative Approach. San Francisco, Calif: Chandler.

BouchaRD, T.J. (1976). Field Research Methods: Interviewing, Questionnaires, Participant Observation, Systematic Observation, Onubstrusive Measures. En Dunnette, M.D. Handbook of Industrial and Organizational Psychology. Chicago. Rand McNally.

BOWERS, D.G. (1968). Organizational Control in an Insurance Company. En Tannenbaum, A.S. (Ed.) Control in Organizations Nueva York: McGrawHill.

DUNCAN, R.B. (1976). The ambidextrous organization: designing dual structures for innovation. En Kilmann, R.H. et al. (Ed.). The Management of Organization Design. Nueva York: Elsevier: North-Holland.

FILLEY, A.C., House, R.J., KeRR, s. (1976). Managerial Process and Organizational Bebavior. Glenview, Ill.: Scott-Foresman.

FINEBERG. H.V. (1985). Technology Assesment: Motivation, Capability and Future Directions. Medical Care, 23, 663-671.

French, J.R. y RAven, B. (1959). The Bases of Social Power, en Cartwright, D. (Ed.): Studies in Social Power, Ann Arbor: University of Michigan Institute for Social Research.

GOUdelaCH, P. y TeTzSCHNER. H. (1976). Measurement of Influence in Organizations: Critique of the Control Grph Method. Acto Sociológica, 19, 49-63.

HaGe, J. (1965). An Axiomatic Theory of Organizations, Administrative Science Quarterly, 10: 289-320.

JENNERGREN, L.P. (1981). Decentralization in Organizations. En Nystrom, P.C. y Starbuck, W.H. (Ed.) Handbook of Organizational Design. Nueva York: Oxford U. Press.

KATZ, D. y KAHN, R.L. (1966). The Social Psychology of Organizations. Nueva York: John-Wiley.

KHANDWALA, P.N. (1977). The Design of Organizations. Nueva York: Harcourt, Brace: Jovanovich.

LAWLER, E.E. (1976). Control Systems in Organizations. En Dunnette, M.D. Handbook of Industrial and Organizational Psychology. Chicago. Rand McNally.

LORSCH, J.W. y MORSE. J.J. (1978). Organizations and their Members: A Contigency Approach. Nueva York: Harper and Row.

MintnzBerg, H. (1984). La Estructuración de las Organizaciones. Barcelona: Ariel.

Overton, P., SchNeCK, R. y Haziett. C.B. (1977). An Empirical Study of the Technology of Nursing Subunits. Administrative Science Quarterly, 22, 203-219.

PEIRO, J.M. (1984). Psicología de la Organización. Madrid: UNED.

Perez QuINTnNA, F. (1987). Evaluación Organizacional de un Hospital. Tesis Doctoral no publicada. Universidad de La Laguna.

PRESTHUS, R.V. (1962). The Organizational Society. Nueva York: Knopf.

PRICE, J.L. (1968). Organizational Effectiveness. Homewood, Ill.: Scott-Foressman.

RABBIE, J.M. y VAN OOSTRUM, J. (1984). Environmental Uncertinty, Power, and Effectiveness in La. boratory Organizations. En Stephenson, G.M. y Davis, J.H. (Ed.) Progress in Applied Social Psychology, 2: $207-261$. 
RAI, G.S. (1985). Control Structure and Conflict in Public Agencies. Administration in Social Work, 9, 75-87.

TANnenbaum, A.S. (1968). Control in Organizations, Nueva York: McGrau-Hill.

VAN de VEN. A.H. y FERRY. D.L. (1980). Measuring and Assesing Organizations. Nueva York: John Wiley.

WEBER, M. (1979). Economía y Sociedad, Segunda edición en español. México: Fondo de Cultura Económica.

ZFY.FerReIL, M. (1979). Dimensions of Organizations: Environment, Context, Structure, Process and Performance. Sta. Mónica, Cal.: Goodyear Publishing Company. 